\title{
The doctor's PDA and Smartphone handbook: medical records
}

\author{
Chris Paton ${ }^{1} \quad$ Mohammad Al-Ubaydli²
}

J R Soc Med 2006;99:183-184

This is the fifth in a series of extracts from a forthcoming book by M Al-Ubaydli and C Paton. The website [www.rsmpress.co.uk/bkpda.htm] includes video tutorials to accompany this text.

The ability to store patient records on a handheld computer is perhaps the feature that clinicians want the most, yet it also is perhaps the hardest to provide. If you are buying a device for yourself simply so that you can use it for medical records, then you should abandon the purchase and save your money.

Let us explain why we say this. It is relatively easy to store information about your patients in your personal database. Buy HanDBase, create your forms and start entering patient data. The difficulty comes when you have to share information with other clinicians. Beaming may be enough for small teams, but for any more than five clinicians, you will need to synchronize your devices with a central computer ... and that requires the help and support of the IT department.

The practice of medicine means that you almost always have to share information with your colleagues. Whether you do it in the paper notes or your institution's electronic medical records system, you must document what you do to the patients so your team can provide appropriate care.

Thus, if you decide that you do not want to share the patient notes on your device because of the complexity of involving the IT department, you will have to duplicate your writing: once for your device and again for the institution's notes. You will soon get tired of this; we are all too busy to be duplicating work.

On the other hand, in institutions around the world, and increasingly with the support of national governments, IT departments are switching to electronic medical records. In many cases, handheld computers are part of that switch - or at least they are planned as the next stage. In such institutions, the doctors will be provided with handheld computers as part of good clinical care.

If you are in such an institution - congratulations, make use of your good fortune and enjoy your equipment!

If you are not, then you can change the institution and convince its decision makers of the investment, but this is a

'Bewdley, Worcestershire, UK; ${ }^{2}$ Bethesda, MD, USA

Correspondence to: Dr Mohammad Al-Ubaydli

E-mail: me@mo-md long process and requires sustained effort. In the meantime, do not buy a handheld computer for yourself in the hope that you will get the support for medical records software. It will not come soon.

Of course, you should still buy the device for all the other advantages, and we hope that we have convinced you of their value. The rest of this article will discuss the options you have as you move your institution to electronic medical records.

\section{MEDICAL RECORDS SYSTEMS FOR INDIVIDUALS AND SMALL TEAMS}

The easiest way to begin managing your patient's records at the start is to buy software like Patient Tracker [www.patienttracker.com], which includes a handheld computer version and a $\mathrm{PC}$ version. This makes it easy to enter most of a patient's information at your clinic desk but to add more details with the handheld computer at the patient's home or to read those details on the device while away from your desk. The data are encrypted.

The software is designed for American clinicians and is ideal for small practices. It is not suitable for general practitioners in the UK, as more than $95 \%$ of their practices already have an electronic medical records system - and their complementary handheld computer versions are better. For doctors in hospitals in the UK that do not yet have a such a system, however, Patient Tracker could be a useful purchase for each small team.

If for any reason you do not like Patient Tracker's interface, however, you cannot modify it. This is why so many clinicians like HanDBase: each team can create the exact forms they need for their workflow. The effort of designing and implementing the forms is not trivial though and will take some time for experimentation.

Whatever you decide, one risk to think about is losing data. You can consider buying a backup SD card for your handheld from companies like MDM [www.gomdm .com] — for around $\$ 50(£ 29)$, the card allows a complete backup of your device. Should you lose the data for any reason, you can restore it within a few minutes after inserting the card into the device. More dangerous is losing the device itself through theft or forgetfulness. This is why encryption is so important. 


\section{BESPOKE MEDICAL RECORDS SYSTEMS}

With the financial and managerial support of your institution, you can consider a medical records system tailored to your team's needs. Scotland has wonderful examples of this, as the government has standardized data formats (which means that medical software designers can more easily share patient data between their products) and has provided budgets for software investments and target dates for implementation. Unlike the government in England, however, Scotland's government has not been involved in micromanaging implementation.

This has led to many new companies creating innovative products and tailoring them to clinicians' needs. For example, Extramed [www.extramed.co.uk] and Kelvin Connect [www.kelvinconnect.com] both created software that allowed hospital clinicians to read information on their patients during the ward round. The information is synchronized automatically between the handheld computer on the ward round, the PCs of the nurses in the medical assessment unit and the workstations of the radiologists in their offices.

What is interesting about these examples is the new working habits that they support and the planning that the team must go through. For example, in Lanarkshire, the software is a small part of the innovation of the night time hospital emergency care teams. A small team of two nurses and five doctors triages and treats the hospital's inpatients overnight. In the morning, the nurses provide a report on each patient they treated, because it was the nurses that prescribed many of the drugs and the nurses that had the handheld computers.

For these habits, the doctors, nurses, managers and software developers worked together and decided on how to best to proceed for their patients. When you understand the information in this book, you will be one of the best people in your institution to help guide the decision-making for handheld computers. Find out about the committees involved in such planning and contribute to them.

\section{OFF-THE-SHELF SYSTEMS}

Even if you cannot contribute to the design of a custom system for your team, you can still play an important role if your institution chooses an off-the-shelf medical records system. This is happening in England, where the government has designated specific medical records system providers for each region. Furthermore, in many institutions, including the practices of all general practitioners in England, complete medical records systems already exist. You will not be able to switch to a different provider, but you still can buy the handheld computer version of their software. For example Inchware the existing products of EMIS, iSoft, Torex and Vision in the UK, while IDX and McKesson in the USA also have had their own mobile software for several years.

Even after the purchasing decision has been made, and even if the product is fixed and not customized, you still will play a crucial role. For example, you can take part in the early testing. This will mean that you can advise on the rate of deployment - which wards should come first — and will be ideally placed to provide training for your colleagues.

Such work is good for your career. For many clinicians, such satisfying work has prompted a switch to medical informatics as a specialty. In the end, of course, it will be the patients that benefit. Medical errors are many, expensive and dangerous. To reduce these and improve care governments around the world are investing billions of dollars in solutions. Electronic medical records are a vital part of these, and handheld computers are already making their mark. We hope that these articles help you bring the benefits to your patients.

\section{Clinical vignette of medical records}

Impressed by the work of Dr Cochrane in her general practice surgery, Dr Snow bought a handheld computer. It greatly improved his organization, and they designed a database to store audit information about their patients with diabetes for audits.

Soon, their seven colleagues wanted to make the same investment. In a practice meeting, they decided that each doctor should buy their own device to suit their tastes. Because the practice had an EMIS medical records system, the clinicians with Palm Powered devices could use the EMIS PDA software, while those with Pocket PCs could get EMIScompatible PDA software from Inchware.

This allowed Dr Snow to take the records of the patients he would see on a home visit, refer to the prescriptions and past medical history while with the patient and make notes on the visit on the handheld computer. Once back in the practice, the new notes would synchronize back to the central computer and become available to the PCs and PDAs of the other doctors and nurses.

The software did not do everything the team required, however, so Dr Snow wrote a business case for the purchase of custom software to monitor the progress of patients with cardiological conditions and diabetes. He received funding from his primary care trust and found a small company in the area. They collaborated on the design of the software and made sure that it integrated into the existing EMIS system.

The product was so well suited to the management of the patients' conditions that he was able to coauthor several papers that documented the improved control of hypertension, and several practices in the surrounding area also invested in the software.

By this time, Dr Snow was hooked on the benefits of handheld computer for good clinical practice. He started a long-distance course in medical informatics, supported by a scholarship from his primary care trust, and searched for other areas in which he could improve patient care. 